\title{
Applications of Distributed and Parallel Computing in the Solvency II Framework: The DISAR System
}

\author{
Gilberto Castellani and Luca Passalacqua \\ Sapienza, Università di Roma, Dipartimento di Scienze Statistiche, \\ Viale Regina Elena, 295, I-00161 Roma, Italy \\ \{gilberto.castellani, luca.passalacqua\}@uniroma1.it
}

\begin{abstract}
We address computational problems deriving from Solvency II compliance in the context of Italian life insurance. Solvency II requires insurance undertakings to perform market consistent valuation of technical provisions and continuous monitoring of risks. We examine the case of profit sharing policies with minimum guarantees, which is the most diffused type of life policy in Italy. Market consistent valuation of the complex cash flows generated by these contracts entails modelling of management actions and the use of numerical techniques in a stochastic framework, typically Monte Carlo simulation on a fine grained time grid. Fulfillment of the subsequent highly-demanding computational tasks is possible only by implementing valuation procedures in parallel and distributed architectures. In this work we introduce DISAR, a Solvency II compliant system designed to work on a grid of conventional computers, and discuss its performances.
\end{abstract}

\section{Introduction}

The European Directive 2009/138 (Solvency II) Dir-09] requires insurance undertakings to evaluate technical provisions in a market-consistent way and to measure the Solvency Capital Requirement (SCR) with the Value-at-Risk approach (confidence level $=99.5 \%$, unwinding period $=1$ year).

Moreover, insurance undertakings "shall have in place an effective risk-management system comprising strategies, processes and reporting procedures necessary to identify, measure, monitor, manage and report, on a continuous basis the risk, at the individual and at an aggregated level, to which they are or could be exposed, and their interdependencies" [Dir-09, art. 44]. It is possible to identify at least five relevant areas that the risk-management system should cover: 1 underwriting and reserving; 2 - asset-liability management; 3 - investment; 4 liquidity and concentration risk management; 5 - risk-mitigation techniques.

The implications and requirements introduced by the Directive become particularly compelling when the undertaking calculates technical provisions and SCR using an "internal model" Dir-09, art. 112]. The internal model is a system used by the undertaking to assess risks and determine the overall solvency needs, 
ensuring the quality standards indicated by the Directive and subject to the approval of the national supervisory authority [Dir-09, art. 112-127]. In addition, the new requirements will have strong impact on the IT function responsibilities, which, instead of being restricted to hardware and software solutions, shall be extended to include processes and monitoring of performance and standards.

In such complex scenario, we shall introduce and discuss DISAR (Dynamic Investment Strategy with Accounting Rules), a computational system designed for the monitoring of portfolios of "profit sharing" Italian life insurance policies with minimum guarantees, linked to "segregated funds". Notice that systems like DISAR are required also when the undertaking computes the SCR using the "standard formula", as presently defined by the Committee of European Insurance and Occupational Pensions Supervisors (CEIOPS) in the Quantitative Impact Study 5 (QIS5) C-10].

\section{Life Insurance Policies in Italy}

Profit sharing policies with minimum guarantees are one of the most popular contracts in the Italian life insurance market. In this type of policies benefits provided to the insured are periodically adjusted depending on the return of a dedicated fund, the so-called segregated fund (in Italian gestione separata) 1 .

As reference example of the profit sharing mechanism, we consider a single premium pure endowment contract, written at time 0 , for a life aged $x$, with term $T$ years and initial sum insured $Y_{0}$. In this case, the benefit $Y_{T}$ paid by the insurer if the policyholder is alive at time $T$ is determined by incrementing each year the insured sum by a fraction of the interest earned by the insurer in the segregated fund where the premium is invested. Specifically,

$$
\begin{gathered}
Y_{T}=Y_{0} \cdot \prod_{k=1}^{T}\left(1+\rho_{k}\right), \text { where } \\
\rho_{k}=\frac{\max \left\{\min \left\{\beta I_{k} ; I_{k}-\eta\right\}-i ; \delta^{c}\right\}}{1+i}
\end{gathered}
$$

is the so-called readjustment rate, $\beta \in(0,1]$ is the participation coefficient, $I_{k}$ is the annual rate of return of the segregated fund in year $[k-1, k], \eta$ is the minimum annual rate retained by the insurance company, $i$ is the technical rate, and $\delta^{c}$ is the minimum guaranteed annual cliquet rate.

The market consistent valuation of the policy must be performed in a stochastic framework where uncertainties are of actuarial and financial type. The valuation principles and the methodological approach are detailed in [DM-05]. The core of market consistent valuation is the fact that value of the benefits at time $t=0, V_{0}\left(Y_{T}\right)$, can be expressed as the expected value of the payoff at time $t=T$,

\footnotetext{
${ }^{1}$ At the end of year 2009 the Italian Supervisory Authority listed 386 segregated funds, belonging to 76 insurance companies, with the overall amount of statutory reserves summing up to about $65 \%$ of the total life reserves.
} 
weighted by a suitable state-price deflator. Assuming independence between actuarial and financial uncertainty, the expectation can be factorised. Finally, the expectations can then be rewritten with a change of measure, employing the so-called risk-neutral probability measure $Q$. Under $Q$ prices measured in units of the value of the money market account are martingales. Accordingly,

$$
V_{0}\left(Y_{T}\right)=\mathbf{E}^{Q}\left[\frac{Y_{T}}{e^{\int_{0}^{T} r_{u} d u}} \mid \mathcal{F}_{0}\right]{ }_{T} p_{x}=\bar{Y}_{T} \mathbf{E}^{Q}\left[\prod_{k=1}^{T}\left(1+\rho_{k}\right) e^{-\int_{0}^{T} r_{u} d u} \mid \mathcal{F}_{0}\right],
$$

where $r_{t}$ is the instantaneous intensity of interest rate determining the value of the money market account, $\mathcal{F}_{t}$ is the filtration containing the information about financial events, $T_{T} p_{x}$ is the risk-neutral probability that an individual aged $x$ will persist for $T$ more years (lapse included) and $\bar{Y}_{T}=Y_{0} p_{x}$ is the actuarially expected benefit. Notice that both $\rho_{t}$ and $r_{t}$ are $\mathcal{F}_{t}$-adapted random variables.

A closer inspection of the payoff of the policy in eq. (11) shows that it includes embedded options, whose underlying is the segregated fund return. The presence of the options can be made explicit by expressing $V_{0}\left(Y_{T}\right)$ using either a put or a call decomposition [DM-05, p. 91]:

$$
V_{0}\left(Y_{T}\right)=B_{0}+P_{0}=G_{0}+C_{0},
$$

where $B_{0}$ is the value of a risky investment (base component) and $P_{0}$ that of a put option; $G_{0}$ is the value a guaranteed investment and a $C_{0}$ that of a call option, or - in the words of the Directive - the policy guaranteed benefits and its future discretionary benefits.

For this type of policies the fund return is typically defined by "book accounting rules" which offer several strategic handles to control the return of the segregated fund and play a key role in the management strategy of the fund itself. Due to the complexity of the payoff and the management actions involved, the valuation processes are performed numerically using Monte Carlo simulation on a fine grained time grid. Fast evaluations are hindered by at least six different factors: 1 - the maturity of policies (about 100 years); 2 - the control of the investment and accounting strategy; 3 - the computing time required by Monte Carlo simulation; 4 - the large number of policies involved (typically at the million level); 5 - the large number of segregated funds owned by each undertaking; 6 - the large number of securities composing each segregated funds.

\section{The DISAR System}

DISAR is a system composed by a DataBase Management System and by several calculation engines. The methodological asset-liability management (ALM) framework in which DISAR has been designed is detailed in CDMP-05, 2 .

\footnotetext{
${ }^{2}$ It is a well established framework embedding all principles of modern finance, rooted in the first works on the market consistent valuation of this type of policies, that date back to 1994 [DM-94] and acknowledging the guidelines suggested in "New Math for Life Actuaries" B-02] and in DM-05].
} 
The theoretical framework and the "high technological" infrastructure allow DISAR to meet the requirements needed for the approval of internal models, i.e. use test Dir-09, art. 120], statistical quality standards (art. 121), calibration standards (art. 122), profit and loss attribution (art. 123), validation standards (art. 124), documentation standards (art. 125).

DISAR is able to provide (inter alia): the market value of the policies, the net asset value (NAV) of the ALM portfolio and the corresponding components (base, call, put and guaranteed); the Value of Business In Force (VBIF); the overall SCR and its components (interest rate, equity, mortality, etc.).

\subsection{Management Actions}

The management of the policy portfolio is modelled defining an asset-liability management ALM strategy, i.e. a set of rules for the actions to be undertaken by the insurance company until run-off of all policies. The strategy affects:

1. the portfolio of outstanding policies (including expenses);

2. the segregated funds, composed by the assets defining the reference index;

3. an external fund, composed by all remaining assets;

4. a bank account, controlling liquidity positions;

5. the subordinated loan capital.

The set of operations that are performed, either monthly or at the end of each accounting year, include:

1. cash-flow hedging: defines how benefits and expenses are paid;

2. technical provision and solvency margin calculation: it is performed at the end of each accounting year;

3. company capital strategy: defines how capital is allocated from the company to shareholders (and vice versa) and how debt is created/cancelled on the company balance sheet; it is performed at the end of each accounting year;

4. fund allocation: defines how capital is allocated between the segregated fund, the external fund and the bank account.

5. asset portfolio management: defines the asset allocation strategies for the segregated fund and the external fund.

The strategy components are governed by algorithms driven by a set of parameters and embody several constraints. For example, the asset portfolio management is defined by creating a classification scheme (asset class) for all possible assets and fixing a range of values for the relative contribution of each class (in terms of value, duration, etc.). Similarly, a target return for the segregated fund can be constrained to be constant or indexed to a financial yield prevailing on the market at that date (e.g. the swap rate for a given maturity).

Since the quantities constrained by the strategy assume values that depend on the trajectory of simulated market prices, the constraints should be periodically monitored along the trajectory making proper readjustments when needed. The monitoring frequency of the constraints strongly affects the overall computing time since it is required to repeat the valuation of a large set of contracts with very long maturities. 


\subsection{Reduction of Computational Complexity}

DISAR achieves a reduction of the computation complexity of the valuations by:

1) decomposing the overall valuation into an actuarial valuation and an ALM valuation as done in eq. (3): all actuarially expected cash flows $\bar{Y}$ are computed first and used later as an input for the ALM valuation;

2) performing scenario generation separately from the actuarial and ALM valuation; this decoupling allows to use exogenous reference trajectories; moreover, the simulation of the trajectories can be easily parallelised;

3) performing on the liability side a decomposition of the contracts, followed by an aggregation of elementary contracts; these are obtained by decomposing derivatives in baskets of simpler contracts; this operation is fully informationpreserving and is different from the traditional model-point technique, where information may be lost;

4) sub-dividing the evaluations to be performed by processing and assembling "atomic units" or elementary elaboration blocks (EEB), each corresponding to a given segregated fund, for a given set of parameters.

The above operations allow the implementation of DISAR in a distributed and parallel computing environment.

\subsection{Sources of Uncertainty}

Two main types of uncertainty are considered: actuarial risks such as mortality/longevity risk, surrender risk (lapses), expense risks and financial risks, such as interest rate risk, equity risk, property risk, credit risk (default and spread risk), currency risk, inflation risk.

Actuarial and financial risks are assumed to be independent. Moreover, actuarial risks are assumed to be independent between each other, while financial risks are possibly correlated. Table 1 reports a list of the main risk drivers with a corresponding model used for the valuation. The list is not exhaustive since in DISAR it is possible to choose between different models: for example credit risk can be modeled either with deterministic credit spreads (as in the "standard formula" QIS5 approach) or with a suitable stochastic model, as in DS-99. Ultimately, the financial uncertainty is described by a vector $\mathbf{Z}$ of Brownian motions, e.g. $\mathbf{Z}=\left\{Z_{t}^{p}, Z_{t}^{r}, Z_{t}^{B}, Z_{t}^{c, j}, Z_{t}^{e, i}\right\}$, and a correlation matrix $\boldsymbol{\Sigma}_{Z}$.

The number of sources of uncertainties is computationally relevant when computing the capital requirement. For each source of uncertainty $s$ the $\mathrm{SCR}_{s}$ is determined as the impact of a specified scenario on the NAV of the undertaking. Two scenarios are considered: one where the source of uncertainty is stressed up to the reference confidence level, and another where the stress is in the opposite direction. For example, for interest rates, let $\Delta_{t}$ be the NAV central value, $\Delta_{t}^{u, d}$ the NAV stressed up and down values; then

$$
\mathrm{SCR}_{\text {int.rate }}=\max \left\{\Delta_{t}-\Delta_{t}^{u} ; \Delta_{t}-\Delta_{t}^{d} ; 0\right\}
$$


Table 1. Financial models

\begin{tabular}{|c|c|c|}
\hline risk driver & evolution & model \\
\hline $\begin{array}{l}\text { consumer prices } \\
\text { expected inflation }\end{array}$ & $\begin{array}{l}d p_{t}=y_{t} p_{t} d t+\sigma_{p} p_{t} d Z_{t}^{p} \\
y_{t}=y_{\infty}+\left(y_{0}-y_{\infty}\right) e^{-\alpha_{y} t}\end{array}$ & $\begin{array}{l}\text { lognormal model } \\
\text { deterministic }\end{array}$ \\
\hline $\begin{array}{l}\text { nominal risk-free int. rates } \\
\text { nominal risk-free disc. factor }\end{array}$ & $\begin{array}{l}d r_{t}=\alpha\left(\gamma-r_{t}\right) d t+\rho \sqrt{r_{t}} d Z_{t}^{r} \\
v(t, s)=A(s-t) e^{-B(s-t) r_{t}}\end{array}$ & $\begin{array}{l}\text { CIR model } \\
\text { CIR model }\end{array}$ \\
\hline risky nominal disc. factors & $v^{j}(t, s)=A^{j}(s-t) e^{-B^{j}(s-t) \eta_{t}^{j}}$ & DS model \\
\hline credit spreads & $d \eta_{t}^{j}=\alpha_{j}^{c}\left(\gamma_{j}^{c}-\eta_{t}^{j}\right) d t+\rho_{j}^{c} \sqrt{\eta_{t}^{j}} d Z_{t}^{c, j}$ & DS model \\
\hline real risk-free rates & $x_{t}=r_{t}-\left(y_{t}-\sigma_{p}^{2}\right)$ & stoch. Fisher eq. \\
\hline $\begin{array}{l}\text { equity benchmark } \\
\text { equity prices }\end{array}$ & $\begin{array}{l}d B_{t}=B_{t} \mu_{B} d t+B_{t} \sigma_{B} d Z_{t}^{B} \\
d S_{t}^{i}=S_{t}^{i} \mu_{S}^{i} d t+S_{t}^{i}\left(\beta^{i} \sigma_{B}\right) d Z_{t}^{B}\end{array}$ & $\begin{array}{l}\text { Black-Scholes model } \\
\text { CAPM }\end{array}$ \\
\hline exchange rates & $d C_{t}^{i}=C_{t}^{i} \mu_{C}^{i} d t+C_{t}^{i} \sigma_{C}^{i} d Z_{t}^{e, i}$ & lognormal model \\
\hline
\end{tabular}

For each segregated fund the number of EEB to be performed is then:

$$
N_{b}=1 \text { (best estimate) }+2 \times \# \text { sources of uncertainty. }
$$

Each elementary block can be processed independently from the other, which allows a trivial parallelisation of the valuation of the different $\mathrm{SCR}_{s}$. Finally, a dedicated EEB performs the consolidation of the $\mathrm{SCR}_{s}$ into the overall SCR.

\section{The Computing Environment}

DISAR works over a grid of conventional computers. Each node of the grid hosts a service that activates the computing engine upon request. The components of the system are the following.

1. A Database Server, hosting a Relational DataBase Management System;

2. A Master Server, hosting the Disar Master Service (DiMAS), that receives the primary requests from the Clients, defines the elementary elaboration blocks, estimates the complexity of the elaborations, establishes the elaboration schedule, distributes the elementary requests to the processing units and monitors the process. If necessary, the Master Service is also able to distribute the data needed for the evaluations and to collect the results and to write them into the Database. In addition, the Master Server hosts also the Disar Consolidation Service (DiConS) that performs the aggregation of the individual results at higher levels.

3. A set of Computing Units: each unit hosts the Disar Engine Service (DiEngS) that manages the Disar Actuarial Engine (DiActEng) and the Disar Alm Engine (DiAlmEng). The Disar Engine Service executes a single run of either one of the two engines. It may write the results directly to the Database or return them to the Master Server. 
Table 2. Computing times. No ordering/Ordered refers to the index of complexity.

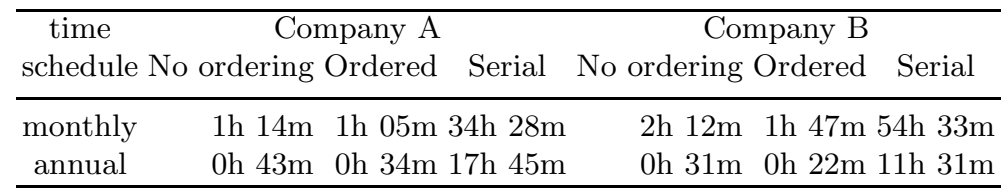

4. A set of Clients, each hosting the Disar Interface (DIINT) that allows to set computational parameters and monitors the progress of the elaborations. Moreover, the DiInT allows to start DiConS directly and gives direct access to the outputs.

\section{DISAR Performances}

DISAR performances were investigated on two (stylised) Italian life insurance companies. Company A (B) has a portfolio of about 5 (1) mln policies referred to 30 (30) segregated funds. The number of policies linked to the same segregated fund ranges from few hundreds to one million (to hundreds of thousands). The number of representative policies - that is the policies having equal insurance parameters (same age, sex, etc.) is about $0.35 \mathrm{mln}(0.4 \mathrm{mln})$. In both cases the maximum time horizon of the policies is 109 years.

The computing grid is composed of 7 heterogeneous computing units for a total of 32 cores. The Relational DataBase Management System is Oracle server $10 \mathrm{~g}$. Connections between units are provided by a LAN with a 1 Gbit HUB switch. The total number $N_{\text {tot }}$ of processed EEB per company was

$$
N_{\text {tot }}=N_{s}[\underbrace{\left(1+2 n_{a}\right)}_{\text {DiACTENG }}+\underbrace{\left(1+2\left(n_{a}+n_{f}\right)+1\right)}_{\text {DiAlMENG }}]+\underbrace{1}_{\text {DiConsEnG }}
$$

where $N_{s}$ is the number of segregated funds, $n_{a}$ is the number of sources of actuarial uncertainty and $n_{f}$ is the number of sources financial of uncertainty and we let DiAlmENG perform an additional forward scenario used to compute the intrinsic and time value of the embedded options (see [CDMP-05], p. 22 for details). In particular, we let $n_{a}=2$ (mortality + lapses) and $n_{f}=2$ (interest rate + equity), which implied a total of $N_{t o t}=451$ EEB per company, of which 150 are of actuarial type and 300 of ALM type, and 1 the (final) consolidation elaboration. 5000 Monte Carlo simulations were used for each EEB. Stressed up/down scenarios correspond to the $99.5 \%$-quantile.

In general the optimal scheduling is driven mainly by the differences in complexity of the jobs and by the differences in computing power of the CPUs. In this application the ordering in complexity has been made using an heuristic index of complexity, computed on the basis of the time horizon of the evaluation, the number of representative policies times the number of elementary contracts, the 
Table 3. Reduction achieved

\begin{tabular}{cccccc}
\hline time & \multicolumn{3}{c}{ Company A } & \multicolumn{3}{c}{ Company B } \\
schedule Ordered & Serial & Reduction & Ordered & Serial & Reduction \\
\hline monthly & $1 \mathrm{~h} 05 \mathrm{~m} 34 \mathrm{~h} 28 \mathrm{~m}$ & 31.8 & 1h $47 \mathrm{~m} 54 \mathrm{~h} 33 \mathrm{~m}$ & 30.6 \\
annual & $0 \mathrm{~h} 34 \mathrm{~m} 17 \mathrm{~h} 45 \mathrm{~m}$ & 31.3 & 0h $22 \mathrm{~m} 11 \mathrm{~h} 31 \mathrm{~m}$ & 31.4 \\
\hline
\end{tabular}

number of assets and asset classes composing the segregated fund and the number of risk drives. The results obtained are not fully optimal since the ordering does not take into account the differences in CPU computing power.

Table 2 reports computing times for the two Companies in 4 different hypotheses: with a monthly/annual time schedule of the ALM strategy and with a casual/ordered submission of the jobs. Computing time is all inclusive; input/output Database access time is estimated to be about $6 \mathrm{~min}$. (5 min.) for Company A (B). Finally, Table 3 shows that, when the jobs are submitted in order of increasing complexity, the reduction factors achieved are almost equal to the number of CPUs used, so that the system is almost optimal.

\section{Conclusions and Outlook}

DISAR has proven to be able to monitor portfolios of profit sharing policies with minimum guarantees as required by the Solvency II Directive. The grid architecture adopted for DISAR is effective, easy to implement and easy to scale. With a small number of non specialised nodes, typical computing times are at most of a couple of hours.

Since the most time consuming jobs are those processed by the ALM engine and involve Monte Carlo calculations, a further improvement of the system is achievable by parallelising the simulations. In fact, it has already been shown that Monte Carlo evaluation in a similar problem scales linearly with the number of CPU's in a "traditional" parallel architecture [CDMP-09]. A promising evolution under study is to use a mixed GPU/CPU architecture where the GPUs are used to speed up the generation of the Monte Carlo sample paths and the conventional CPUs are used whenever a large quantity of data is needed in the calculation.

\section{References}

[B-02] Bühlmann, H.: New Math for Life Actuaries. Astin Bulletin 32(2) (2002)

[C-10] CEIOPS: QIS5 Technical Specifications (July 2010)

[CDMP-05] Castellani, G., De Felice, M., Moriconi, F., Pacati, C.: Embedded Value in Life Insurance. Working paper (2005)

[CDMP-09] Corsaro, S., De Angelis, P.L., Marino, Z., Perla, F., Participating life insurance policies: an accurate and efficient parallel software for COTS clusters. Computational Management Science (2009) doi: 10.1007/s10827-009-0100-0, ISSN 1619-697X 
[Dir-09] Directive 2009/138/EC of the European Parliament and of the Council of 25 November 2009 on the taking-up and pursuit of the business of Insurance and Reinsurance. Official Journal of the European Union (2009)

[DM-94] De Felice, M., Moriconi, F.: Un modello per la progettazione e la valutazione di polizze indicizzate e rivalutabili. Rapporto per l'INA, Roma (1994)

[DM-05] De Felice, M., Moriconi, F.: Market Based Tools for Managing the Life Insurance Company. Astin Bulletin 35(1), 79 (2005)

[DS-99] Duffie, D., Singleton, K.: Modeling Term Structures of Defaultable Bonds. Review of Financial Studies 12(4), 687 (1999) 